\title{
Private Topic or Public One: On Topic Sentence Quality
}

\author{
LIU Dong-hong \\ Central China Normal University, Wuhan, China
}

\begin{abstract}
Topic effect on L2 writing has long been an issue of controversy. This study was carried out to investigate the influence of topic type on the quality of topic sentence. The participants, 150 college students at three different proficiency levels, were asked to write both on a private topic and a public topic. The results show that the participants on the whole wrote better on the public topic, and their proficiencies can be differentiated by the quality of their topic sentences. The public topic was conducive to the production of complete English discourse structures, as well as the generation of informative and predictive topic sentences. Moreover, the results also reveal the dynamic nature of contrastive rhetoric.
\end{abstract}

Keywords: topic effect, topic sentence, EFL (English as a Foreign Language) writing, proficiency

\section{Introduction}

Much second language writing research has focused on topic effects (Hinkel, 2009; Bonzo, 2008; Sullivan, 2007; Winfield \& Barnes-Felfeli, 1982; QIN \& GU, 2011). But most of the studies are interested in the use of certain syntactic and lexical features in L2 writing. The scant amount of research on the effects of public and private topics has produced inconclusive results. Hamp-Lyons and Mathia (1994) and Hinkel (2009) have found that public topic facilitates English writing while Reed and Vandett (1985) have different results. Therefore, more research is needed to examine the impact of such essay topics.

Although topic sentence in L2 writing has been widely studied for decades, yet the major concern of the researchers is the indirectness of paragraphs against different cultures, such as Kaplan (2000), Hind (1987), and Connor (1996), or the placement of topic sentences (CHEN, 2011; WU, 2003). However, the research on the quality of topic sentences is scant. What is more, whether topic type has influence on the production of topic sentences is in need of research. To contribute to this domain, the present study focuses on topic types and makes an exploration into their effects on topic sentence quality.

\section{Topic Effects on L2 Writing}

Most of the studies on the effects of private topics and public ones reveal that public topics facilitate English writing. Hamp-Lyons and Mathia (1994) studied the scores of 8,000 essays written on 64 prompts. Their results showed that essays on private topics received lower scores than those on public topics since many L2 learners had less exposure to personal than academic topics and hence failed to use skillfully personal experience as evidence. Hinkel (2009) analyzed modal verb uses in L1 and L2 essays on five private and public topics, and found that the

LIU Dong-hong, professor, Ph.D., School of Foreign Languages, Central China Normal University. 
frequency rates of possibility and ability modal verbs are less topic-dependent than obligation and necessity modals in the L2 essays. The study concluded that public topics elicit fewer differences between L1 and L2 essays than topics in which personal experiences have to be drawn on because the former is less culturally-dependent. However, Reed and Vandett (1985) get different results. They analyzed the essays of 44 college freshmen in basic writing classes and found that the essays about group-phenomenon events had lower quality scores because of syntactic confusion than the essays about individually experienced events, although they contained more words per clause.

Generally speaking, the research mentioned above has examined whether topic type has an effect on the frequency of use of certain syntactic and lexical features in L2 writing. Few studies have investigated topic effects on topic sentences written by L2 students at different proficiency levels. More research is needed to identify such impact of essay topics.

\section{Research Questions}

Topic sentence in this study refers to the claim supporting the thesis statement. Many students, especially the middle group and the high group, tended to put each claim and its supporting sentences in a separate paragraph. In view of the inconsistent placement of the claim, the term "segmented discourse" (Asher, 1994) is used instead of "paragraph", to refer to an argument, i.e., a claim together with its supporting sentences. Since a topic sentence serves not merely as a claim but also a summary of its supporting sentences, what really count is its lexical appropriateness and discourse relations between the topic sentence and its supporting sentences, which can be categorized into rhetorical mode. Since in the Chinese style of paragraph writing topic sentence is considered unnecessary and unimportant, the Chinese participants might transfer their L1 writing style. In view of that, discourse structure is also investigated in the study of topic sentence. To cover all these elements, an umbrella term "topic sentence quality" is employed. The research questions deal with two aspects.

(1) Does topic type exert influence on the quality of the topic sentence on the whole?

(2) Do the students at different proficiency level vary in the quality of topic sentence?

\section{Research Methods}

The essays analyzed in this study were written by 150 college and university students, of whom 50 were second-year English majors (high group), 50 first-year English majors (middle group), and another 50 first-year non-English majors (low group). All of the participants had already learned English exposition. The high group had already passed Test for English Major-Band 4 (TEM-4), and the middle group was preparing for the coming TEM-4 of the next year. And the low group was preparing for the College English Test-Band 4 (CET-4 for non-English major) which was much easier than TEM-4. In this study, they were asked to write two essays with prompts after class as usual homework and were permitted to consult dictionaries. The essay prompts were modeled on standardized tests such as TEM-4 and CET-4, for the purpose of eliciting essays in the rhetorical mode of exposition and informing a general audience. One essay had public topic "The Benefits of Travelling Abroad" and the other had private one "I Prefer to Work in..." The public topic was relatively remote to the students' life since nearly none of them had traveled abroad or considered the travelling, although they knew much about the world from internet and TV. But their hot topic and major concern had been their future job since 
they entered the college and university.

Based on the discourse topic theory (Asher, 1994), coherence theory (Giora, 1997), and Monroy-Casas's (2008) guidelines, the topic sentence quality was divided into five ranks (LIU, 2012), roughly as follows:

Rank 0: No topic sentence for a segmented discourse.

Rank 1: No supporting sentence follows the topic sentence. Or the supporting sentences cannot support the topic sentence at all.

Rank 2: The topic sentence is not fully supported by the other sentences.

Rank 3: The topic sentence can summarize the supporting sentences but it is too general, and the adjectives are too vague.

Rank 4: The topic sentence encapsulates appropriately the supporting sentences.

The average quality of topic sentence in each essay could be obtained by adding up the scores of all the topic sentences and then dividing the result by the total number of arguments. In addition, the median frequency rates of each rank in the essays were also calculated. The author of the paper evaluated the topic sentences of the compositions for two times, with an interval of two months. The final score of each topic sentence is the average of the two scores. In data analysis, non-parametric statistical comparisons of the topic types were employed because neither the frequency rates nor the generalization rate was normally distributed.

\section{Results}

Table 1 demonstrates that topic type exerts an influence on the topic sentence quality on the whole and that the topic sentences of the essays on the public topic have higher quality than those on the private topic (2.54 > $2.24)$ and the difference is statistically significant $\left(-2.98^{* * *}, p=0.003\right)$. To be specific, each group performed better in the essays on the public topic than on the private one. However, only high group shows significant difference ( $p=0.03$ ) but in the other two groups the difference between the two topics is weak. In addition, it can be noted in Table 2 that the topic sentence quality in the two essays could differentiate the students of different proficiencies because the difference among the three groups is statistically significant. The mean scores of the low group in the two essays are around 1.5, those of the middle group are around 2.5 and those of the high group are above 3 .

Table 1

Influence of Topic Type on the Topic Sentence Quality

\begin{tabular}{|c|c|c|c|c|c|c|c|c|}
\hline \multirow{2}{*}{ Topic type } & \multicolumn{2}{|c|}{ Low group } & \multicolumn{2}{|c|}{ Middle group } & \multicolumn{2}{|c|}{ High group } & \multicolumn{2}{|c|}{ Total } \\
\hline & Mean & $S D$ & Mean & $S D$ & Mean & $S D$ & Mean & $S D$ \\
\hline Private topic & 1.34 & 0.71 & 2.34 & 1.04 & 3.05 & 1.04 & 2.24 & 1.17 \\
\hline Public topic & 1.57 & 0.74 & 2.66 & 1.01 & 3.40 & 0.75 & 2.54 & 1.12 \\
\hline$Z$ value & \multicolumn{2}{|c|}{$-1.26, p=0.21$} & \multicolumn{2}{|c|}{$-1.56, p=0.12$} & \multicolumn{2}{|c|}{$-2.13^{*}, p=0.03$} & \multicolumn{2}{|c|}{$-2.98^{* *}, p=0.003$} \\
\hline
\end{tabular}
Note. ${ }^{*} p<0.05,{ }^{* *} p<0.01$.

Table 1 only displays a global result. The specific results can be seen from Table 3 which shows the proportion of all kinds of ranks. Rank 1 and rank 2 were combined to be considered as a whole because they both denoted the case of "failing to summarize" which had a radical difference from rank 0 (i.e., incomplete discourse structure without topic sentence) and from rank 3 (i.e., the topic sentence can summarize the supporting sentences 
but it is too broad). In Table 3, rank 0 occupies the least of the proportion in both the high group (3\%) and the middle group (5\%), and the second least is rank 3. But it is opposite in the low group: Rank 0 and rank 4 have nearly the same percentage higher than rank 3 . Moreover, the gaps among the three groups in ranks $1 \& 2$ and in rank 4 are wide: In the high group, rank 4 takes up the majority of the proportion while ranks $1 \& 2$ dominate in the low group; but the dominance of ranks $1 \& 2$ becomes less salient in the middle group. In a general term, the sequence of the frequency rates is: ranks $1 \& 2$ (52\%) > rank 4 (35\%) > rank 3 (8\%) > rank 0 (5\%). Figure 1 displays the dynamic nature of the four ranks.

Table 2

Difference of Proficiency in Terms of Topic Sentence Quality

\begin{tabular}{llllllll}
\hline \multirow{2}{*}{ Topic type } & \multicolumn{3}{c}{ Low group } & \multicolumn{3}{c}{ Middle group } & \multicolumn{3}{c}{ High group } & \multirow{2}{*}{$\mathrm{X}^{2}$} \\
\cline { 2 - 8 } & Mean & $S D$ & Mean & $S D$ & Mean & $S D$ & \\
\hline Private topic & 1.34 & 0.71 & 2.34 & 1.04 & 3.05 & 1.04 & $57.44^{* * *}, p=0.000$ \\
Public topic & 1.57 & 0.74 & 2.66 & 1.01 & 3.40 & 0.75 & $68.69^{* * *}, p=0.000$ \\
\hline
\end{tabular}

Note. ${ }^{* * *} p<0.001$.

Table 3

Proportions of Different Ranks of Quality

\begin{tabular}{llcll}
\hline Groups & Rank 4 (\%) & Rank 3 (\%) & Ranks 1 \& 2 (\%) & Rank 0 (\%) \\
\hline Low group & 8 & 4 & 81 & 7 \\
Middle group & 31.5 & 13.5 & 50 & 5 \\
High group & 64 & 6.5 & 26 & 3 \\
Total & 35 & 8 & 52 & 5 \\
\hline
\end{tabular}

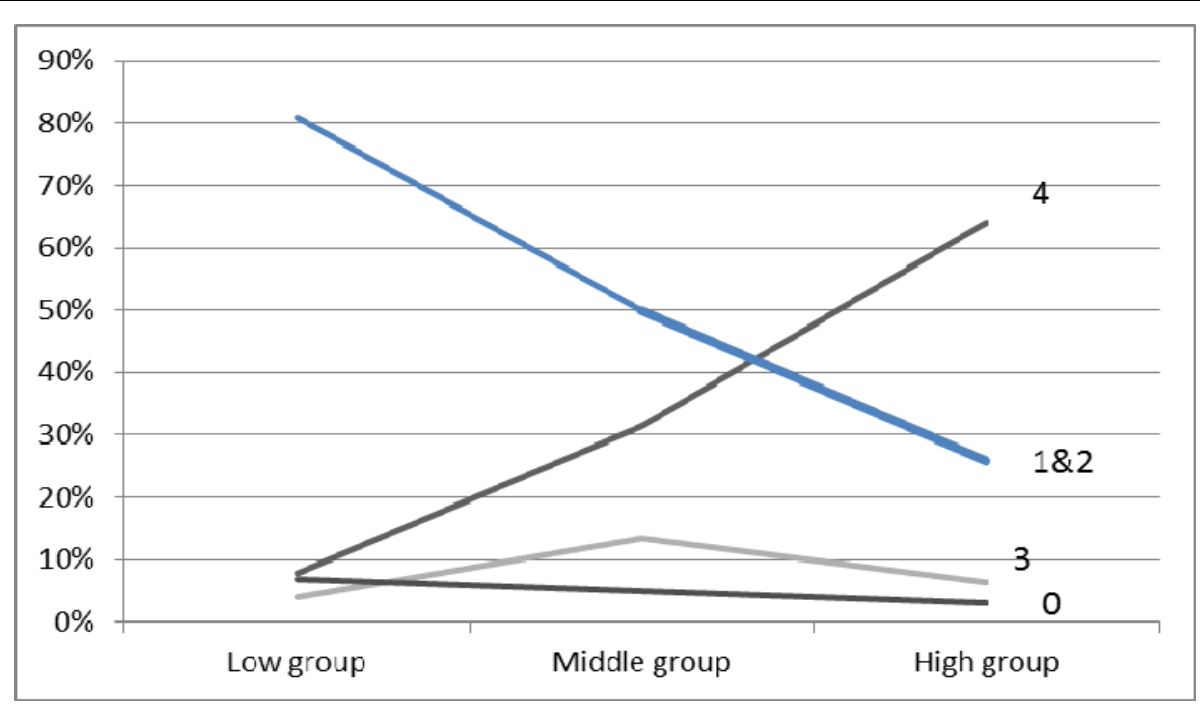

Figure 1. Dynamic nature of the four ranks.

\section{Discussion}

Topic effects can be seen in Table 1 in that the students, especially the high group, wrote better on the public topic than on the private one. The lower mean quality score (2.24) in the private topic essays means the students could barely summarize their segmented discourses by topic sentences or develop their topic sentences at a full length. But the mean score in the public topic essays is nearly 3 (2.54), which means that the topic sentences 
could function as summarizers, that is to say, the segmented discourses were developed well around the topic sentences. A contributing factor may be the English writing instruction. In China, most of the students at the tertiary level taking their English writing course write more on public topics since the ultimate goal of the training is TEM4 or CET4. It can be speculated that just because all of the students had learned expository writing skills for standardized tests which had a preference to public topics, the public topic in this study might activate their knowledge and skills and it was easier to apply them to such a topic. However, the private topic offered them relatively more freedom from choosing the topic to deciding the content. Just because they had so many choices about where to work and so many reasons to support their thesis statements, some students failed to fully develop their claims and switched to new reasons abruptly and unconsciously. For Example (1), when the readers read the topic sentence they expect that why or how "life in big cities is much more convenient" is going to be explained. But there is only one incomplete sentence supporting the claim and the third sentence switches to "comfort". The inadequate development of the segmented discourse undermines the topic sentence quality. That example also reveals the transfer of the Chinese writing style. In Chinese writing instruction, the students are asked to "use fewer words to express the same ideas; therefore, at least some of the writer's ideas have to be written suggestively” (X. WANG, 1994, p. 225). Consequently, their paragraphs are not fully developed to support the topic sentences. Just as Hinkel (2002) has found in his study, the Chinese students failed to provide specific information.

Example (1) The last but not the least, life in big cities is much more convenient. Fast public transport, a variety of goods to choose from and plenty of public places for enjoyment. Above all, the perfect health care system and other insurance make you live in great comfort.

Moreover, some students were eager to express their ideas so much so that they took an emotional style without providing any topic sentence or supporting sentences. In Example (2), "If I can, I prefer to work in Xiangyang - my hometown, forever, with you” is not the topic sentence but the thesis statement. The thesis statement was repeated for several times. That is consistent with one of the Chinese writing skills—resorting to emotion and appealing to the readers. The Chinese books for writing such as Textbook for Argumentative Writing by T. S. WANG (2006) and New Strategies for Argumentative Writing by YU (2009) advocate that writers may persuade the readers with emotion and many ways of resorting to emotion are proposed. As X. WANG (1994, p. 214) has pointed out that English writers state their ideas in words while Asians write with emotion as readers need to feel or guess the ideas from the writing so as to appreciate it.

Example (2) If I can, I prefer to work in Xiangyang — my hometown, forever, with you.

Let's ignore the so-called dreams or struggle. Just stay in our hometown, our familiar hometown, where we can call out the names of each street. We know each of our neighbors. When we want to play majiang, karaoke or go for shopping, no more than half an hour, every of us will be there.

If I can, I prefer to work in Xiangyang, forever, with you.

We can accompany with our parents and stick to our hometown when we feel bored. We can go to Wuhan for fun, which will just take a few hours' driving. During festival, we can visit each of our friends, have a party and if who dares not to come, we just need to take a taxi and pull him out.

In general, the participants performed better when given a public topic. The result corroborates Hamp-Lyons and Mathia (1994) and Hinkel (2009) from a different perspective of L2 writing. 
Apart from that, the topic sentence quality in both the private topic essays and the public ones can differentiate the learners at different proficiency levels. The ability of discourse organization and coherence is a part of language performance. And language performance improves with proficiency levels. On the other hand, the measurement of the topic sentence quality proves to be objective and reasonable.

\section{Conclusion}

The results of this study demonstrate that topic sentence production is affected by the essay topic to a great extent and the degree of topic sentence quality can differentiate the learners of different proficiencies. It is not because the private topic was more culturally-dependent as proposed by Hinkel (2009), but because the higher familiarity and more freedom debilitate the L2 learners to use the English way of discourse organization. Specifically, the influence is mainly on the discourse structures and the appropriateness of generalization.

Although Kaplan (2000), X. WANG (1994), and Monroy-Casas (2008) contend that linear rhetoric is in no way superior to non-linear rhetoric, and what exists is a different way of presenting ideas across cultures, yet in EFL (English as a Foreign Language) teaching, it is still expected that the students (especially those future teachers in normal universities) to acquire the culture as well as the language since the two parts are inseparable. As is shown in this study, for the Chinese learners of English familiar private topics cannot facilitate English writing since their practice in writing is mainly oriented to academic tests. Against the background of writing instruction in China, private topics seem to be more difficult to write on. However, preparing the students for the standard tests should not be the ultimate goal of English writing instruction at tertiary level. And English writing instruction should not neglect free private topics. The training may begin with easier public topics, and then move to private ones. The difficulty of private topics lies in the competition of too many ideas. So another recommendation for English writing instruction is to put on restrictions on the private topics at the very beginning, and then to give the learners more and more freedom gradually as well as more reminding or requirement of the English way of writing.

\section{References}

Asher, N. (1994). Reference to abstract objects in discourse. Dordrecht: Kluwer Academic Publishers.

Bonzo, J. D. (2008). To assign a topic or not: Observing fluency and complexity in intermediate foreign language writing. Foreign Language Annals, 41(4), 722-736.

CHEN, W. J. (2011). An investigation of topic sentences in Chinese students' argumentative essays: A multidimensional probe. Kristianstad University, Kristianstad. Retrieved from hkr.diva-portal.org/smash/get/diva2.../FULLTEXT01

Connor, U. (1996). Contrastive rhetoric: Cross-cultural aspects of second-language writing. Cambridge: Cambridge University Press.

Giora, R. (1997). Discourse coherence and theory of relevance: Stumbling blocks on search of a unified theory. Journal of Pragmatics, 27, 17-34.

Hamp-Lyons, L., \& Mathia, S. (1994). Examining expert judgements of task difficulty on essay tests. Journal of Second Language Writing, 3, 49-68.

Hinds, J. (1987). Reader versus writer responsibility: A new typology. In U. Connor \& R. B. Kaplan (Eds.), Writing across languages: Analysis of L2 texts. Reading, MA: Addison-Wesley.

Hinkel, E. (2002). Second language writers’ text. Mahwah, NJ: Lawrence Erlbaum Associates.

Hinkel, E. (2009). The effects of essay topics on modal verb uses in L1 and L2 academic writing. Journal of Pragmatics, 41, 667-683.

Kaplan, R. B. (2000). Contrastive rhetoric and discourse analysis: Who writes what to whom? When? In what circumstances?. In S. Sarangi \& M. Coulthard (Eds.), Discourse and social life (pp. 82-101). Edinburgh: Pearson Education (Longman). 
LIU, D. H. (2012). Predictive language and global coherence in English learners' compositions. Foreign Language Teaching, 4, 53-57.

Monroy-Casas, R. (2008). Linearity in language: Rhetorical-discursive preferences in English and Spanish in the light of Kaplan's model. International Journal of English Studies, 8(2), 173-189.

QIN, Z. X., \& GU, Q. Y. (2011). Topic familiarity and the application of cohesive devices in Chinese L2 writers' written discourses. Journal of Xi'An International Studies University, 1, 95-98.

Reed, W. M., \& Vandett, N. M. (1985). Initiation versus intensification: The effect of topic-type on the writing performance of basic writers. The 8th Annual Meeting of the Eastern Educational Research Association, Virginia Beach, VA.

Sullivan, A. S. (2007). The effects of topic choice and prompts on student writing and student attitudes about writing (Unpublished dissertation). University of Massachusetts Lowell, MA.

WANG, T. S. (2006). Textbook for argumentative writing. Changsha: Hunan University Press.

WANG, X. (1994). Writing concepts in Chinese writing instruction. Issues in Applied Linguistics, 5(2), 211-229.

Winfield, F. E., \& Barnes-Felfeli, P. (1982). The effects of familiar and unfamiliar cultural context on foreign language compositions. Modern Language Journal, 66, 373-378.

WU, J. (2003). An investigation of the characteristics of argumentative essays written by college students: The usage of topic sentences in paragraphs and in texts. Foreign Language Research, 91(3), 35-42.

YU, D. X. (2009). New strategies for argumentative writing. Shanghai: Shanghai Culture and Arts Press. 